\title{
Avaliação da adesão ao tratamento de pacientes com diabetes mellitus e seus fatores associados
}

\author{
Adherence to the diabetes mellitus treatment evaluation and associated factors
}

Evaluación de la adhesión al tratamiento de pacientes con diabetes mellitus y sus factores asociados

Ana Paula Morais Corrêa Machado1, Ana Carine Gomes Santos ${ }^{1}$, Karine Kerolly Almeida Carvalho', Manuela Pierotte Luz Gondim¹, Naiara Pinto Bastos ${ }^{1}$, João Vítor Souza Rocha ${ }^{1}$, Olívia Abreu Versiani ${ }^{1}$, Maria Thereza Meira Araujo', Fernando Gonçalves Brasil Filho', Júlia Colares Moreira $^{3}$, Fernanda Araújo Sá ${ }^{3}$, Bruna Afonso Lopes Lima ${ }^{3}$, Isabelle Almeida Pessoa ${ }^{3}$, João Pedro Paulino Ruas ${ }^{1}$, Karina Andrade de Prince ${ }^{1 *}$.

\section{RESUMO}

Objetivo: Verificar a adesão ao tratamento da Diabetes Mellitus tipo 2 (DM2)em pacientes do Núcleo de Atenção à Saúde e Práticas Profissionalizantes (NASPP), Montes Claros-MG. Métodos: Estudo de campo transversal com abordagem quantitativa descritiva através de formulários específicos. São eles: Formulário Sociodemográfico, Questionário clínico MAT (Questionário de Medida de Adesão aos Tratamentos) e Questionário de Avaliação do Autocuidado adaptado. Amostra selecionada de 43 pacientes de ambos os sexos assistidos no setor de Endocrinologia do NASPP. Os dados foram submetidos à análise descritiva, através de média, desvio-padrão e frequência, adotando-se nível de significância $p<0,05$. Resultados: $A$ maior parcela amostral pertencia ao sexo feminino (62,8\%), na faixa etária de 50-59 anos (44,2\%); 69,8\% da amostra alcançou a meta terapêutica; $53,8 \%$ da amostra não tomou o medicamento alguma vez, sendo, $32,5 \%$ por se sentir melhor, $26,9 \%$ por se sentir pior e $50 \%$ porque o medicamento acabou. Houve significância estatística da relação entre dieta e exercício físico no controle glicêmico. Conclusão: Apesar de $100 \%$ da amostra ser aderente ao tratamento farmacológico, 30,2\% dos pacientes não alcançaram a meta de controle dos seus níveis glicêmicos. Sendo assim, destaca-se a importância da associação entre o tratamento farmacológico, a realização de dieta balanceada e prática de exercícios físicos para atingir a meta glicêmica.

Palavras-chave: Diabetes Mellitus tipo 2, Qualidade de vida, Cooperação, Adesão ao Tratamento.

\footnotetext{
ABSTRACT

Objective: To analyze the adherence to the treatment of DM2 in patients of the Núcleo de Atenção à Saúde e Práticas Profissionalizantes (NASPP), Montes Claros-MG. Methods: Transversal field study with quantitative descriptive approach through specific forms. They are: Sociodemographic Form, Clinical Questionnaire MAT (Questionnaire of Measure of Adherence to the Treatments) and Self-Care Assessment Questionnaire adapted. Selected sample of 43 patients of both sexes attended in the sector of

${ }^{1}$ Faculdades Integradas Pitágoras de Montes Claros (MG), Brasil.

${ }^{2}$ Faculdades Unidas do Norte de Minas (FUNORTE), Montes Claros (MG), Brasil.

*E-mail: karina.prince@bol.com.br
} 
Endocrinology of NASPP. The data were submitted to descriptive analysis, by mean, standard-deviation and frequency, adopting a level of significance $p<0.05$. Results: The majority of the sample eas female $(62,8 \%)$, in the age of $50-59$ anos $(44,2 \%) ; 69,8 \%$ reached therapy goals; $53,8 \%$ of the sample did not take the meds anytime,being that $32,5 \%$ claimed to feel better, $26,9 \%$ to feel worse and $50 \%$ because meds were over. There was statistical significance in the relation between diet and exercises on glycemic control. Conclusion: Althought $100 \%$ of the sample has showed farmacological treatment adherence, $30,2 \%$ of the patients has not reached their glycemic control goal. Therefore, stands out the importance between the associatiom of farmacological therapy, balanced diet and the practice of exercises in order to achieve the glycemic control.

Key-words: Diabetes Mellitus type 2, Quality of life. Treatment Adherence, Compliance.

\section{RESUMEN}

Objetivo: Verificar adhesión al tratamiento de la DM2 en pacientes del Núcleo de Atención a la Salud y Prácticas Profissionalizantes (NASPP), Montes Claros-MG. Métodos: Estudio de campo transversal con abordaje cuantitativo descriptiva a través de formularios específicos.Son ellos: Formulario Sociodemográfico, Cuestionario clínico MAT(Cuestionario de Medida de Adhesión a los Tratamientos) y Cuestionario de Evaluación del Autocuidado adaptado.Muestra seleccionada de 43 pacientes de ambos sexos asistidos en el sector de Endocrinologia del NASPP. Los datos fueron sometidos al análisis descriptivo, a través de media, desvío-normalizado y frecuencia, adoptándose nivel de significancia $p<0,05$. Resultados: La mayor cuota amostral pertenecía al sexo femenino (62,8\%),en el rango etária de 50-59 años (44,2\%);69,8\% de la muestra alcanzó la meta terapéutica;53,8\% de la muestra no tomó el medicamento alguna vez siendo,32,5\% por sentirse mejor,26,9\% por sentirse peor y $50 \%$ porque el medicamento acabo. Hubo significancia estadística de la relación entre dieta y ejercicio físico en el control glicêmico. Conclusión: A pesar del 100\% de la muestra ser adherente al tratamiento farmacológico,30,2\% de los pacientes no alcanzaron la meta de control de sus niveles glicêmicos.Siendo así, se destaca la importancia de la asociación entre el tratamiento farmacológico, la realización de dieta balanceada y práctica de ejercicios físicos para alcanzar la meta glicêmica.

Palabras clave: Diabetes Mellitus tipo 2, Calidad de vida, Cumplimiento, Adherencia al Tratamiento.

\section{INTRODUÇÃO}

O diabetes mellitus (DM) é uma doença crônica considerada um problema grave de saúde pública no Brasil e no mundo devido sua alta incidência e prevalência. A projeção é que, em 2030, mais de 300 milhões de pessoas sejam portadores de DM. A classificação do DM segue a Organização Mundial da Saúde (OMS) e a Associação Americana de Diabetes (ADA), baseando- se na etiologia, incluindo quatro classes: DM tipo 1 (DM 1), DM tipo 2 (DM 2), DM gestacional (DMG) e outros tipos de DM. $O$ risco de desenvolver DM 2 conta com fatores de risco modificáveis que consistem no sobrepeso, obesidade central, na Hipertensão Arterial Sistêmica (HAS), glicemia plasmática alterada, dislipidemias, hábitos alimentares inadequados, sedentarismo e no tabagismo, e os fatores de risco não-modificáveis que são a idade e o antecedente familiar de diabetes (FERREIRA, et al., 2011; CARRERA CA e MARTINEZ-MORENO JM, 2016).

Atualmente são três os métodos aceitos para o diagnóstico de DM com utilização da glicemia: sintomas de poliúria, polidipsia e perda ponderal de peso acrescidos de glicemia casual; glicemia de jejum e glicemia de 2 horas pós-sobrecarga de $75 \mathrm{~g}$ de glicose. Considera-se, ainda, valores de hemoglobina glicada (HbA1c) para diagnóstico de DM, sendo esta mais útil para avaliar controle da glicemia (SBD, 2015).

O tratamento do DM visa manter o controle metabólico e compõe-se de terapia não medicamentosa e medicamentosa, sendo a primeira relacionada às mudanças de comportamento associadas à adesão a uma 
alimentação saudável, redução de peso e monitorização dos níveis glicêmicos e realização de atividade física com frequência e tempo individualizados para cada paciente com o objetivo de redução de peso. A terapia medicamentosa é baseada no uso de hipoglicemiantes orais ou insulina (GOMES LC, et al., 2011).

A Sociedade Brasileira de Diabetes (2015) recomenda que o início do tratamento medicamentoso no paciente diabético seja logo ao diagnóstico, com o uso de agentes hipoglicemiantes orais, associado às mudanças no estilo de vida. Outros hipoglicemiantes ou insulina são utilizados caso a glicemia do paciente não atinja os níveis glicêmicos preconizados.

Entende-se como adesão ao tratamento a coincidência entre o comportamento do paciente com a orientação médica no que se refere ao uso da medicação prescrita e mudanças no estilo de vida (VILLAS BOAS, et al., 2014).

Os principais fatores associados à adesão ao tratamento são estudados há muitos anos, visto que a mesma é influenciada por variáveis multifatoriais. Estas foram identificadas, assim, em mais de duzentas e categorizadas em quatro grandes grupos de fatores implicados na adesão ao tratamento medicamentoso: aqueles atribuídos ao paciente, à relação profissional-paciente, ao esquema terapêutico e à doença, quando excluído o fator da falta de acesso à medicação (SILVA I, et al., 2006; TURCATTO HG, et al., 2009).

No entanto, a falta de adesão ao tratamento é um desafio encontrado pelos profissionais de saúde, uma vez que, apesar de desempenharem papel vital no controle das doenças crônicas, a responsabilidade pelo tratamento, reside, em última instância, no doente ou em seus familiares (SILVA I, et al., 2006).

Em relação aos pacientes com $\mathrm{DM} 2$, as dificuldades encontradas para a adesão ao tratamento são fatores como regime medicamentoso complexo (número elevado de drogas e de tipos de medicamentos), maior tempo de doença, uso de insulina e mau controle glicêmico, dieta restritiva e dificuldade para realização de atividade física (FARIA, et al., 2013; FRANCO AJ, et al., 2013).

O DM2 é uma doença crônica de alta morbidade e mortalidade. Desta maneira, segundo Villas Boas e colaboradores (2014), a manutenção de níveis normais de glicemia e hemoglobina glicada deve ser alcançada com a adesão ao tratamento para diminuir o risco de lesões microvasculares e macrovasculares. Dessa forma, quando não controlada a glicemia, este descontrole pode levar o paciente a desenvolver tais complicações, com comprometimento renal, ocular e neuronal, além do aumento do risco para doenças cardiovasculares como infarto agudo do miocárdio e acidente vascular cerebral. (SANTOS AL, et al., 2015).

As pessoas portadoras de DM2 têm um risco aumentado de desenvolver as complicações microvasculares e macrovasculares. Por vezes, os sintomas da doença não são suficientemente intensos ou podem estar ausentes, levando a um grau de hiperglicemia capaz de causar alterações morfológicas ou funcionais, durante um longo período antes de o diagnóstico ser estabelecido (PAIVA C, 2001). Isso ocorre devido ao fato de que o Diabetes Mellitus está associado à disfunção endotelial, que é o marcador mais precoce e conhecido da aterosclerose e alterações da reatividade microvascular estão presentes tanto nesses pacientes quanto em indivíduos com fatores de risco para essa doença (AGUIAR LGK, et al., 2007).

As complicações a longo prazo associadas ao DM2, têm elevado custo econômico: os pacientes diabéticos consomem mais que o dobro dos cuidados de saúde que os não diabéticos, devido, especialmente, ao alto custo do tratamento dessas consequências à má adesão do tratamento (CRUZ SC, 2005).

Apesar do DM2 não apresentar cura, seu controle através de mudanças no estilo de vida e uso correto do tratamento farmacológico diminuem as chances do paciente apresentar tais complicações, e, deste modo, torna-se de fundamental importância para a prática clínica avaliar a adesão destes ao tratamento, reformular a abordagem aos pacientes mais resistentes ao uso de medicamentos e mudanças no estilo de vida, para, assim, alcançar melhores resultados, além de diminuir o custo econômico da doença(SANTOS AL, et al., 2015). 
Desta forma, percebendo-se a importância do tratamento adequado do DM2 na prevenção de complicações, este estudo teve como objetivo avaliar a adesão ao tratamento dos pacientes com DM2 atendidos no Núcleo de Atenção à Saúde e Práticas Profissionalizantes (NASPP), bem como seus fatores associados.

\section{MÉTODOS}

Trata-se de um estudo de campo de caráter transversal com abordagem quantitativa descritiva. A pesquisa foi realizada no setor de endocrinologia do Núcleo de Atenção à Saúde e de Práticas Profissionalizantes (NASPP), pertencente às Faculdades Integradas Pitágoras de Montes Claros, MG.

A amostra foi composta por pacientes de ambos os sexos, com diagnóstico de DM2 e em tratamento ( $\mathrm{n}$ = 43), com boas condições de comunicação e cognição, e maiores de 18 anos. Estes aceitaram participar da pesquisa, assinaram o Termo de Consentimento Livre e Esclarecido e se enquadraram nos critérios de inclusão. O trabalho foi enviado e aprovado pelo Comitê de Ética e Pesquisa das Faculdades Integradas Pitágoras de Montes Claros - MG, sendo o número do parecer 2.002.387.

Os dados foram obtidos primeiramente por meio de uma entrevista com a utilização de um questionário sociodemográfico (desenvolvido pelos pesquisadores), em que foram construídas perguntas para se avaliar idade, sexo, oxupação, grau de instrução e estado marital; um clínico (Medida de adesão ao tratamento MAT) (VILLAS BOAS LC, et al., 2014), e de autocuidado adaptado (TOOBERT DJ, et al., 2000). Posteriormente, foram avaliados os prontuários dos pacientes para verificação dos níveis glicêmicos, através dos valores de hemoglobina glicada.

As variáveis que constituíram o instrumento de coleta foram as seguintes: aspectos sociodemográficos (idade, sexo, ocupação, grau de instrução e estado marital), fatores clínicos - MAT (medicamento em uso, tempo de tratamento, esquecimento de tomada de medicamentos, descuido com o horário de tomada de medicamentos, omissão de tomada de medicamentos por diversos motivos, interrupção da terapêutica por várias razões) e práticas realizadas para o autocuidado (dieta e exercício físico).

Para avaliação da amostra quanto sua adesão ao tratamento este estudo considerou principalmente a medida da hemoglobina glicada, uma vez que apesar de os questionários de autocuidado e o questionário de aderência à medida de tratamento (MAT) utilizarem questões específicas que abrangem as principais atividades de autocuidado do doente diabético, uma resposta positiva já inclui o paciente em aderido ao tratamento, podendo, portanto, superestimar a adesão.

Os dados obtidos foram submetidos a um processo de análise descritiva, através de média, desvio padrão e frequência, que serviu para caracterizar a amostra. As análises estatísticas foram realizadas com auxílio do Statistical Package for the Social Sciences (SPSS Inc., Chicago, EUA), versão 14.0. Inicialmente, foram descritas as frequências simples e as porcentagens das variáveis analisadas. A análise bivariada entre variáveis independentes (sexo, faixa etária, anos de estudo, estado marital, atividade remunerada, tempo de tratamento, tipo de tratamento e autocuidado) e o desfecho (adesão ao tratamento), foi realizada por meio do teste de qui-quadrado de Pearson, adotando-se um nível de significância de $p<0.05$.

\section{RESULTADOS}

Foram avaliados 43 portadores de DM2, acompanhados no serviço de endocrinologia do NASPP. A maioria se encontrava entre 50 e 59 anos (n. $19 / 44,2 \%$ ), possuíam baixa escolaridade - ensino fundamental incompleto (n. $22 / 51,2 \%$ ) e ensino fundamental completo (n. 12/27,9\%), relataram ter companheiro fixo (n. 28 / 65,1\%) e exercem atividade remunerada (n. 28 / 65,1\%). Em relação aos dados clínicos, 33 pacientes estavam em uso de antidiabéticos orais $(76,7 \%)$ e $10(23,3 \%)$ em uso de insulina. Sendo que, 24 pacientes $(55,8 \%)$ estão há mais de 5 anos em tratamento para o DM2 (Tabela 1). 
Tabela 1 - Dados sociodemográficos dos pacientes portadores de DM2

\begin{tabular}{|c|c|c|c|}
\hline & & $\mathrm{N}$ & $\%$ \\
\hline \multirow[t]{2}{*}{ Sexo } & Feminino & 27 & 62,8 \\
\hline & Masculino & 16 & 37,2 \\
\hline \multirow[t]{6}{*}{ Faixa etária } & 30 a 39 & 4 & 9,3 \\
\hline & 40 a 49 & 5 & 11,6 \\
\hline & 50 a 59 & 19 & 44,2 \\
\hline & 60 a 69 & 10 & 23,2 \\
\hline & 70 a 79 & 4 & 9,3 \\
\hline & $>80$ & 1 & 2,4 \\
\hline \multirow{3}{*}{$\begin{array}{l}\text { Anos de } \\
\text { estudo }\end{array}$} & $<5$ anos & 22 & 51,2 \\
\hline & 5 a 9 anos & 12 & 27,9 \\
\hline & 10 out & 9 & 20,9 \\
\hline \multirow{2}{*}{$\begin{array}{l}\text { Estado } \\
\text { marital }\end{array}$} & Companheiro fixo & 28 & 65,1 \\
\hline & Sem companheiro fixo & 15 & 34,9 \\
\hline \multirow{2}{*}{$\begin{array}{l}\text { Atividade } \\
\text { remunerada }\end{array}$} & Sim & 28 & 65,1 \\
\hline & Não & 15 & 34,9 \\
\hline \multirow{6}{*}{$\begin{array}{l}\text { Tipo de } \\
\text { tratamento } \\
\text { Tempo de } \\
\text { tratamento }\end{array}$} & Antidiabéticos orais & 33 & 76,7 \\
\hline & Insulina & 10 & 23,3 \\
\hline & $>1$ ano & 3 & 7,0 \\
\hline & 1 e 2 anos & 6 & 14,0 \\
\hline & 3 e 4 anos & 10 & 23,2 \\
\hline & $>5$ anos & 24 & 55,8 \\
\hline
\end{tabular}

Fonte: Dados da pesquisa, 2016.

De acordo com as metas terapêuticas estabelecidas pela Sociedade Brasileira de Diabetes, a maioria dos pacientes (n. 30 / 69,8\%) alcançaram a meta terapêutica de hemoglobina glicada (Tabela 2).

Analisando a não adesão ao tratamento medicamentoso dos pacientes avaliados, bem como seus motivos, verificou-se que $53,8 \%$ (n.23) deixaram de tomar o medicamento alguma vez. Sendo que, a maioria $(60,86 \%)$ deixou de tomar porque se sentiram melhor ou porque o medicamento acabou $(56,5 \%)$ (Figura 1).

A avaliação do questionário de autocuidado relacionado ao alcance das metas terapêuticas de hemoglobina glicada ( $\mathrm{HbA1C})$ dos indivíduos portadores do DM2 demonstrou que indivíduos que realizam dieta e atividade física por mais vezes na semana obtiveram um maior controle glicêmico. Sendo assim, através deste estudo foram obtidos resultados estatisticamente significativos da correlação entre o controle glicêmico e as variáveis dieta $(p=0,05)$, consumo de frutas e verduras $(p=0,05)$ e realização de exercícios físicos gerais $(0,04)$ e específicos $(p=0,03)$. No entanto, não foi encontrada significância nas demais variáveis (Tabela 3). 
Tabelas 2-Pacientes que alcançaram as metas terapêuticas

\begin{tabular}{|c|c|c|c|}
\hline & & $\mathbf{N}$ & $\%$ \\
\hline \multirow[t]{2}{*}{ Sexo } & Feminino & 21 & 70,0 \\
\hline & Masculino & 9 & 30,0 \\
\hline \multirow[t]{6}{*}{ Faixa etária } & 30 a 39 & 2 & 6,7 \\
\hline & 40 a 49 & 2 & 6,7 \\
\hline & 50 a 59 & 11 & 36,7 \\
\hline & 60 a 69 & 10 & 33,3 \\
\hline & 70 a 79 & 4 & 13,3 \\
\hline & $>80$ & 1 & 3,3 \\
\hline \multirow{3}{*}{$\begin{array}{l}\text { Anos de } \\
\text { estudo }\end{array}$} & $<5$ anos & 15 & 50,0 \\
\hline & 5 a 9 anos & 10 & 33.3 \\
\hline & 10 ou + & 5 & 16,7 \\
\hline \multirow{2}{*}{$\begin{array}{l}\text { Estado } \\
\text { marital }\end{array}$} & Companheiro fixo & 20 & 66,7 \\
\hline & $\begin{array}{l}\text { Sem companheiro } \\
\text { fixo }\end{array}$ & 10 & 33,3 \\
\hline \multirow{4}{*}{$\begin{array}{l}\text { Atividade } \\
\text { remunerada } \\
\text { Tipo de } \\
\text { tratamento }\end{array}$} & Sim & 23 & 76,7 \\
\hline & Não & 7 & 23,3 \\
\hline & $\begin{array}{l}\text { Antidiabéticos } \\
\text { orais }\end{array}$ & 25 & 83,3 \\
\hline & Insulina & 5 & 16,7 \\
\hline \multirow{4}{*}{$\begin{array}{l}\text { Tempo de } \\
\text { tratamento }\end{array}$} & $>1$ ano & 1 & 3,3 \\
\hline & 1 e 2 anos & 6 & 20,0 \\
\hline & 3 e 4 anos & 8 & 26,7 \\
\hline & $>5$ anos & 15 & 50,0 \\
\hline
\end{tabular}

Fonte: Dados da pesquisa, 2016.

Figura 1 - Motivos pelos quais os pacientes deixaram de tomar os medicamentos (hipoglicemiantes orais e insulina) em algum momento do seu curso terapêutico

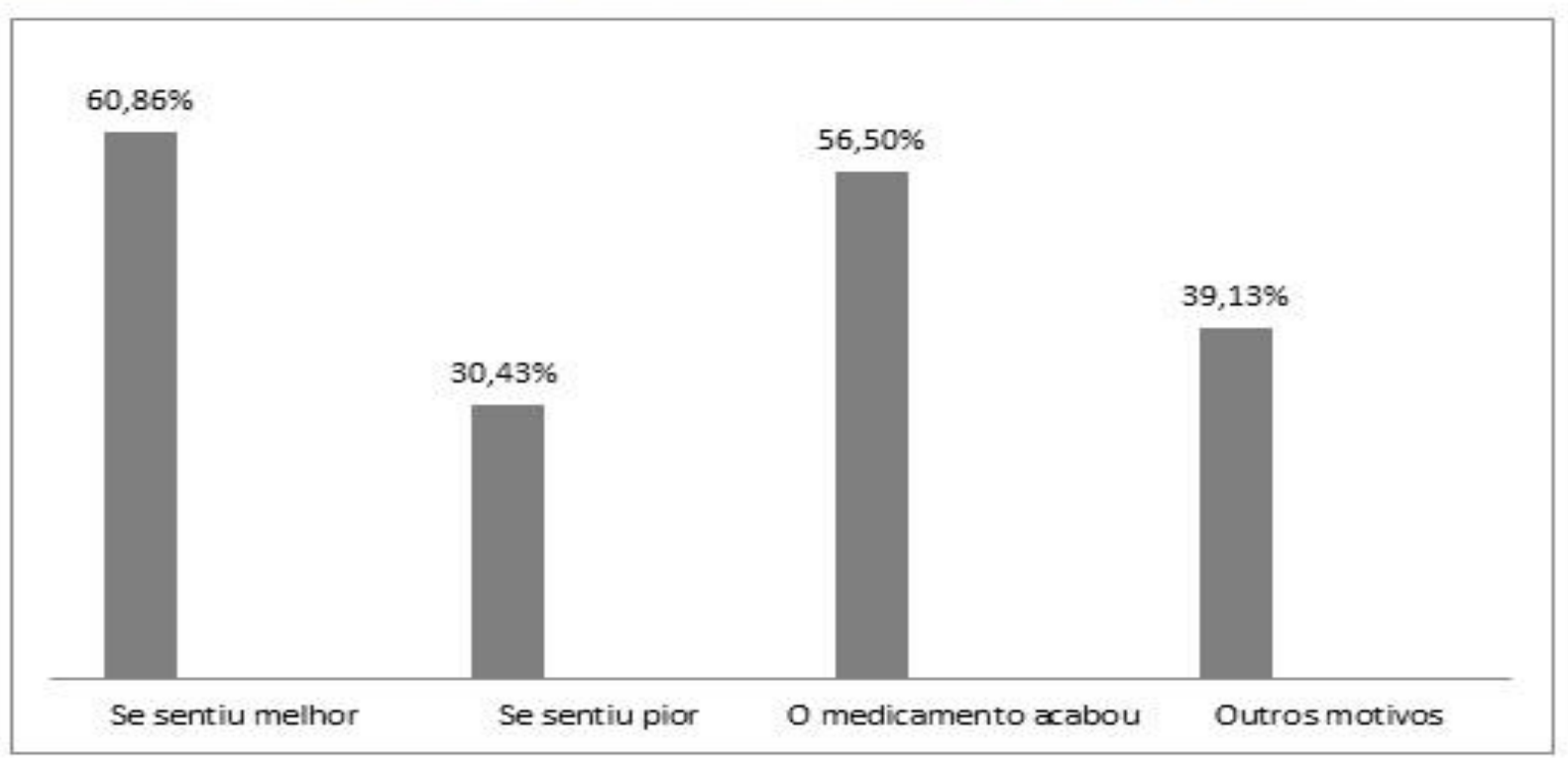

Fonte: Dados da pesquisa, 2016. 
Tabela 3 - Relação entre autocuidado e alcance das metas terapêuticas dos pacientes portadores de Diabetes Mellitus tipo 2

\begin{tabular}{lllll} 
& Mínimo & Máximo & Média & $\mathrm{P}$ \\
\hline $\begin{array}{l}\text { Em quantos dos últimos SETE DIAS seguiu uma } \\
\text { dieta saudável? }\end{array}$ & 1 & 10 & 3,14 & 0,04 \\
\hline $\begin{array}{l}\text { Durante o último mês, QUANTOS DIAS POR } \\
\text { SElmo }\end{array}$ & 1 & 11 & 4,86 & 0,07
\end{tabular}

SEMANA, em média, seguiu a orientação

alimentar, dada por um profissional de saúde

(médico, enfermeiro, nutricionista)?

\begin{tabular}{|c|c|c|c|c|}
\hline $\begin{array}{l}\text { Em quantos dos últimos SETE DIAS comeu cinco } \\
\text { ou mais porção de frutas e/ou vegetais? }\end{array}$ & 0 & 14 & 4,56 & 0,05 \\
\hline $\begin{array}{l}\text { Em quantos dos últimos SETE DIAS não comeu } \\
\text { alimentos ricos em gordura como carnes } \\
\text { vermelhas ou derivados de leite integral? }\end{array}$ & 0 & 13 & 4,25 & 0,09 \\
\hline $\begin{array}{l}\text { Em quantos dos últimos SETE DIAS não comeu } \\
\text { doces? }\end{array}$ & 0 & 17 & 4,6 & 0,20 \\
\hline $\begin{array}{l}\text { Em quantos dos últimos SETE DIAS realizou } \\
\text { atividade física durante pelo menos } 30 \text { minutos? } \\
\text { (minutos totais de atividade contínua, inclusive } \\
\text { andar). }\end{array}$ & 1 & 8 & 3,51 & 0,04 \\
\hline $\begin{array}{l}\text { Em quantos dos últimos SETE DIAS praticou } \\
\text { algum tipo de exercício físico específico (nadar, } \\
\text { caminhar, andar de bicicleta), sem incluir suas } \\
\text { atividades em casa ou trabalho? }\end{array}$ & 2 & 5 & 3,11 & 0,03 \\
\hline
\end{tabular}

Mínimo: 0 dias

Máximo: 7 dias

Fonte: Dados da pesquisa, 2016.

\section{DISCUSSÃO}

O DM2 é uma doença crônica de alta morbidade e mortalidade. O controle metabólico é complexo e depende da implementação efetiva de diversas ações, como controle dietético, exercício físico, acompanhamento adequado do tratamento medicamentoso, entre outros. A obtenção de um controle metabólico adequado do DM evita o início ou retarda a progressão das complicações da doença. É importante, assim, que o paciente diabético atinja a meta terapêutica, que é $\mathrm{HbA} 1 \mathrm{c}$ ao redor de $7 \%$ em adultos e entre 7,5 e 8,5\% em idosos, dependendo do estado de saúde (ARRELIAS CC, et al., 2015; SBD, 2015).

No presente estudo, foi encontrado "p" significativo verificou-se associação entre o controle glicêmico com exercício e dieta. O exercício físico regular e o seguimento do plano alimentar têm grande papel no controle e na prevenção de complicações do DM2. Porém, a prática regular de exercício físico e a adoção de um plano alimentar equilibrado é uma meta difícil de ser alcançada em pacientes idosos e naqueles com comorbidades (FARIA, et al., 2013).

Ao avaliar os dados sociodemográficos, houve maior prevalência DM2 entre pacientes do sexo feminino, na faixa etária de 50 e 59 anos, com baixa escolaridade, que apresentavam companheiro fixo e exerciam atividade remunerada. Em relação aos dados clínicos, a maioria deles utilizavam antidiabéticos orais e estavam há mais de 5 anos em tratamento para o DM2.

O predomínio de DM2 entre as mulheres pode estar relacionado à maior procura destas pelos serviços de saúde, sendo observado, muitas vezes, o homem como provedor da família, o que lhe dificulta a procura pelo atendimento clínico (GRILLO MF e GORINI MC, 2007). No entanto, não há diferença na prevalência de DM2 entre homens e mulheres. 
De acordo com Mendes e colaboradores (2011), os fatores que influenciam o DM ser mais expressivo nas faixas etárias mais elevadas são a escolaridade, o estilo de vida, consumo de álcool e do cigarro, a incidência de outras doenças crônicas. Muitos dos pacientes se recusam a seguir corretamente 0 tratamento, podendo fazer uso de hipoglicemiantes orais e de insulina, no entanto, se negam a fazerem dietas alimentares para perda de peso e a prática de exercícios físicos.

A importância de verificar o grau de instrução do paciente se deve ao fato de que a condição de escolaridade pode dificultar o acesso às informações e trazer menores oportunidades de aprendizagem quanto ao cuidado com a saúde, uma vez que, a maior parcela dos pacientes diabéticos, ao adquirirem conhecimento de sua condição, realizam autocuidado adequado (GRILLO MF e GORINI MC, 2007).

A hemoglobina glicada é um parâmetro importante para o controle glicêmico em pacientes com DM, visto que possui forte relação com o início e progressão das complicações crônicas da doença. Assumindo que pacientes diabéticos com níveis mais baixos de $\mathrm{HbA} 1 \mathrm{c}$ têm melhor qualidade de vida, e são mais aderentes ao tratamento da doença (GUSMAI LF, et al., 2015; HUA X, et al., 2016).

$O$ fato de a baixa adesão estar entre os de maior idade, pode estar associado a fatores inerentes ao envelhecimento. A polifarmácia relacionada aos problemas cognitivos, como o esquecimento, e limitações físicas, como problemas visuais, e até a baixa escolaridade e conhecimento sobre a doença são fortes preditores de não adesão ao seguimento medicamentoso (ARRELIAS CC, et al., 2015).

Quanto ao estado marital, acredita-se que os pacientes com companheiro fixo possuem maior controle metabólico devido ao apoio familiar e o compartilhamento do mesmo espaço físico e do plano alimentar da família (RODRIGUEZ ML, et al., 2015).

Segundo Faria (2009), a falta de compreensão do paciente com relação ao medicamento prescrito pode causar resultados insatisfatórios no tratamento. Quando o portador de DM2 inicia a terapêutica medicamentosa, deve ser avaliada a sua capacidade de entendimento para que suas metas glicêmicas sejam alcançadas. Dessa forma, pode-se associar a esse fator, a menor adesão ao tratamento em pacientes que fazem uso de insulina, como visto neste estudo, uma vez que sua aplicação é de complexidade maior que a ingestão de medicação oral. Há, dessa forma, a necessidade de construção de um processo de reorientação da atenção ao paciente nos serviços de saúde, pois não basta oferecer os medicamentos, é preciso avaliar a forma como vêm sendo utilizados.

Em relação ao tempo de diagnóstico, os resultados mostraram que os pacientes com mais de cinco anos de diagnóstico apresentaram maior adesão ao tratamento medicamentoso, alimentar e exercício físico. Entretanto, a Organização Mundial da Saúde afirma que os portadores de DM2 diagnosticados por um maior período de tempo tendem a apresentar menor taxa de adesão ao tratamento (OMS; FARIA HT, et al., 2013). Apesar de este estudo ter demonstrado que 100\% dos pacientes apresentavam adesão ao tratamento, cerca de $30 \%$ deles não alcançaram a meta terapêutica, demonstrando que houve uma superestimativa de adesão. Uma justificativa para esta discordância de resultados é que parte dos pesquisados eram menos aderentes à medicação deixando de usar os hipoglicemiantes em algum momento ao longo do tratamento.

Os dados do presente estudo corroboram com os do estudo de Santos, Oliveira e Colet (2010), que verificou uma prevalência de 40 a $60 \%$ de pacientes em tratamento que não fazem uso dos medicamentos prescritos adequadamente para determinada doença. Os principais motivos pelos quais os pacientes deixaram de tomar o medicamento foram por se sentirem melhor e pelo medicamento ter acabado. Este fato é constatado também em outros estudos e é explicado por Gomes-Villas Boas, Foss-Freitas, Pace (2014). Segundo os autores, pessoas com DM, especialmente com o tipo 2 da doença, são mais propensos a uma baixa adesão ao tratamento medicamentoso, devido ao caráter assintomático da doença e ausência de queixas físicas levando à crença de que a medicação não é necessária, o que não acontece com as doenças agudas e sintomáticas.

Os pacientes que deixam de tomar a medicação pelo término da mesma, muitas vezes, não têm conhecimento adequado da doença e da importância do uso prolongado da medicação, já que a efetividade 
do tratamento de doenças crônicas depende, essencialmente, de dois fatores: a eficácia do tratamento prescrito e adesão contínua a este, diminuindo as complicações relacionadas à patologia. (ARAÚJO, et al., 2010). Além disso, a disponibilidade da medicação é fator importante, de acordo com o estudo realizado por Mendes et al. (2014), que analisou a disponibilidade de medicamentos em unidades de saúde e concluiu que foi baixa a proporção de unidades com disponibilidade total dos medicamentos chaves em todos os grupos farmacológicos, incluindo antidiabéticos, ainda que com gradiente variável a depender do porte populacional e do próprio grupo farmacológico. Sendo assim, a falta de medicação em unidades de saúde pode atrapalhar a adesão do paciente ao tratamento.

Infere-se, portanto, que este estudo mostra-se como instrumento de avaliação da adesão ao tratamento do DM2 por uma população limitada, contribuindo, apesar do viés apresentado, para demonstrar e reiterar a importância da correta abordagem ao paciente diabético para que este não sofra com complicações futuras da doença. Contribui-se, desta maneira, com os diferentes fatores que podem interferir na adesão ao tratamento da doença e propõe-se modifica-los, a fim de reduzir o índice de morbidade da mesma.

\section{CONCLUSÃO}

Constatou-se, no presente estudo, que, apesar de $100 \%$ da amostra ser considerada aderente ao tratamento, 30,2\% dos pacientes não alcançaram a meta terapêutica de hemoglobina glicada, uma vez que realização de dieta adequada e prática de exercícios físicos também são fundamentais para o bom controle glicêmico. Reitera-se, dessa forma, a importância da instrução ao paciente diabético, quanto ao tratamento não-medicamentoso e medicamentoso, garantindo sua compreensão do estado crônico constituído pelo DM2, a fim de garantir a adesão ao tratamento e evitar as possíveis comorbidades da doença.

\section{REFERÊNCIAS}

1. AGUIAR LGK, VILLELA NR, BOUSKELA E. A Microcirculação no Diabetes: Implicações nas Complicações Crônicas e Tratamento da Doença. Arq. Bras.Endocrinol. Metab., 2007; 51 (2): 204-2011.

2. ARRELIAS C, et al. Adesão ao tratamento do diabetes mellitus e variáveis sociodemográficas, clinicas e de controle metabólico. Acta paul. enferm, 2015; 28 (4): 315-322.

3. CARRERA CA.; MARTINEZ-MORENO JM. Pathophysiology of diabetes mellitus type 2: beyond the duo "insulin resistance-secretion deficit". Revista Nutricion Hospitalaria, 2016.

4. CRUZ, SC. Tratamento não farmacológico da Diabetes tipo 2. Rev. Port. Clin. Geral, 2005; 21: 587-595.

5. FARIA HT et al. Fatores associados à adesão ao tratamento de pacientes com diabetes mellitus. Acta paul. enferm, 2013; 26(3): 231-237

6. FARIA HT et al. Qualidade de vida de pacientes com diabetes mellitus antes e após participação em programa educativo. Revista da Escola de Enfermagem da USP, 2013; 47(2): 348-354

7. FARIA HT et al.. Conhecimento sobre terapêutica medicamentosa em diabetes: um desafio na atenção à saúde. Acta Paulista de Enfermagem, 2009; 22(5): 612-617

8. FERREIRA LT, SAVIOLLI IH, VALENTI VE et al. Diabetes melito: hiperglicemia crônica e suas complicações. Arquivos Brasileiros de Ciências da Saúde, 2011; 36(3): 182-188.

9. FRANCO AJ, HELENO, MG, LOPES AP. Qualidade de vida e controle glicêmico do paciente portador de Diabetes Mellitus tipo 2. Revista Psicologia e Saúde, 2013; 5(2): 102-108.

10. GOMES LC, FOSS MC, PACE AE. Adesão de pessoas com diabetes mellitus tipo 2 ao tratamento medicamentoso. Rev Bras Enferm, 2014; 67(2): 268-73.

11. GOMES LC et al. Adesão à dieta e ao exercício físico das pessoas com diabetes mellitus. Texto Contexto Enfermagem, 2011; 272-279.

12. GONÇALVES TC, DAMASCENO MMC, CAETANO JA. Adesão de diabéticos ao tratamento medicamentoso. Esc Anna Nery Rev Enferm, 2010; 14 (2): 361-367.

13. GRILLO MF, GORINI MC. Caracterização de pessoas com Diabetes Mellitus tipo 2. Rev. Bras. Enferm, 2007; 60(1): 49-54.

14. GUSMAI LF, NOVATO TS, NOGUEIRA, LS. The influence of quality of life in treatment adherence of diabetic patients: a systematic review. Rev. esc. enferm. USP,2015; 49(5):839-846 
15. HUA X, LUNG TW, PALMER A et al. How Consistent is the Relationship between Improved Glucose Control and Modelled Health Outcomes for People with Type 2 Diabetes Mellitus? a Systematic Review. PharmacoEconomics, 2016

16. MENDES LV et al. Disponibilidade de medicamentos nas unidades básicas de saúde e fatores relacionados: uma abordagem transversal. Saúde Debate | Rio de Janeiro,2014; 38: 109-123.

17. PAIVA C. Novos critérios diagnósticos e classificação da diabetes mellitus. Medicina Interna, 2001.7 (4): 234 238.

18. RODRIGUEZ ML et al. Prevalencia y factores asociados a la adherencia al tratamiento no farmacológico en pacientes con hipertensión y diabetes en servicios de baja complejidad. Rev. Fac. Nac. Salud Pública, 2015; 33 (2): 192-199.

19. SANTOS AL et al. Complicações microvasculares em diabéticos Tipo 2 e fatores associados: inquérito telefônico de morbidade autorreferida. Ciência e Saúde Coletiva, 2015; 20(3): 761-770.

20. SILVA I; RIBEIRO JP, CARDOSO H. Adesão ao tratamento da diabetes Mellitus: A importância das características demográficas e clínicas. Revista Referência, 2016; 2.

21. OLIVEIRA JE, VENCIO S. Diretrizes da Sociedade Brasileira de Diabetes: 2014-2015, 2015.

22. TURCATTO HG, ZANETTI ML, HAAS VJ Fatores relacionados à adesão do paciente diabético à terapêutica medicamentosa. Revista Latino-Americana de Enfermagem, 2009.

23. TOOBERT, DJ et al. The summary of diabetes self-care activities measure: results from 7 studies and a revised scale. Diabetes care, 2000.

24. VILLAS BOAS LC, FOSS MC, PACE AE. Adesão de pessoas com diabetes mellitus tipo 2 ao tratamento medicamentoso. Revista Brasileira de Enfermagem, 2014; 67(2): 268-273. 